Artículos Científicos

\title{
Desarrollo de habilidades STEM en media superior como mecanismo para impulsar la continuidad en educación superior: Caso programa Bases de Ingeniería
}

Development of STEM Skills in High School as a Mechanism to Improve Continuity in Higher Education: Case Bases of Engineering Program

Desenvolvimento de habilidades STEM no ensino superior como um mecanismo para promover a continuidade do ensino superior: caso do programa Engineering Bases

Luis Fernando Pantoja Amaro Colegio de Estudios Científicos y Tecnológicos, México pantoja@queretaro.gob.mx https://orcid.org/0000-0001-9839-8851

Juan Manuel Peña Aguilar Universidad Del Valle de México, México juan_manuelp@hotmail.com https://orcid.org/0000-0002-7605-238X

Christian Paulina Mendoza Torres Universidad de Celaya / Universidad Tecnológica Laja Bajío, México pmendoza@udec.edu.mx https://orcid.org/0000-0002-2998-714X 


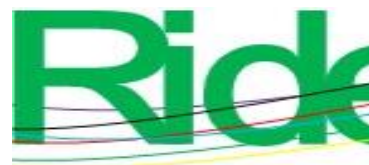

Revista lberoamericana para la Investigación y el Desarrollo Educativo ISSN $2007-7467$

\section{Resumen}

La educación media superior (EMS) en México es ahora considerada parte de la educación obligatoria en el país. Ante esta obligatoriedad el desarrollar una educación que tenga un mayor impacto en el entorno tanto productivo como educativo cobra una mayor importancia. El aprendizaje basado en proyectos y casos prácticos ha demostrado ser una forma de generar un aprendizaje significativo, aunque desarrollarlo con los escasos recursos que se tienen en las instituciones públicas en el país representa un enorme reto. Por otra parte, está comprobado que las habilidades ligadas al enfoque de las ciencias integradas, a saber: ciencia, tecnología, ingeniería y matemática (STEM, por sus siglas en inglés), son muy importantes en la integración de un aprendizaje más activo y con mejor comprensión de los contenidos científicos. En la EMS en México existen el modelo tradicional de educación, la formación técnica y el modelo dual (ahora mediante el modelo mexicano de formación dual); sin embargo, a pesar de que son buenos esfuerzos, están más enfocados en la educación tradicional, en la capacitación técnica o en la resolución de problemas para adquirir experiencia que en desarrollar una habilidad de "ingenio" para la resolución de problemas prácticos pero no enfocados necesariamente al trabajo en una empresa en particular; habilidad que les permita tener facilidad para resolver problemas y enfrentarse mejor a compromisos como hacer frente al estudio de una ingeniería y posteriormente a diferentes tareas de investigación aplicada.

Así, pues, tomando como base el programa Project Lead The Way (PLTW), que se desarrolló en Estados Unidos, el Colegio de Estudios Científicos y Tecnológicos de Querétaro (Cecyteq) desarrolló un programa denominado Bases de Ingeniería, el cual busca desarrollar en los estudiantes de EMS habilidades que les permitan enfrentarse con mayor éxito ya sea tanto a los estudios universitarios de ingeniería como al desarrollo de actividades profesionales en su especialidad técnica en el campo laboral. Este artículo muestra el seguimiento a la eficiencia del programa y la satisfacción que este ha provocado en los estudiantes. Lo anterior con la finalidad de entender cómo aplicar programas especiales que permitan formar talento competitivo a pesar de no contar con un incremento de los recursos materiales disponibles, en un primer comparativo en los planteles Corregidora, Huimilpan, Menchaca, Montenegro, Pedro Escobedo, Querétaro y San Juan del Río.

Palabras clave: aprendizaje basado en proyectos, bases ingeniería, media superior, STEM. 


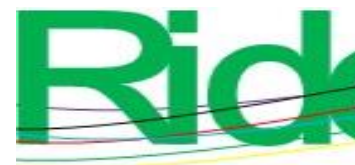

Revista Iberoamericana para la

Investigación y el Desarrollo Educativo

ISSN $2007-7467$

\section{Abstract}

High school in Mexico is now considered part of obligatory education in the country. Before this obligation to develop an education that has a greater impact on the productive and educational environment becomes more important. Learning based on projects and case studies has proven to be a way to generate meaningful learning, although the way to develop it with the scarce resources that are available in public institutions in the country represents an enormous challenge. In higher education in Mexico there is the traditional model of education, technical training and the dual model (now through the Mexican model of dual training); however, although they are good efforts, are more focused on traditional education, in technical training or problem solving to gain experience that in developing a skill of "ingenuity" to solve practical problems but not necessarily focused on work in a particular company; skill that allows them to have ease to solve problems and better deal with commitments such as dealing with the study of an engineering and subsequently with different tasks of applied research. Thus, based on the Project Lead The Way (PLTW), program that was developed in the United States, the Colegio de Estudios Científicos y Tecnológicos de Querétaro (Cecyteq) elaborated a program called Bases de Ingeniería to develop in the students of high school the skills that allow them to face with greater success the university studies of engineering as well as to the development of professional activities in their technical specialty in the labor field. This article shows the follow-up to the efficiency of the program and the satisfaction that it has caused in the students. This to understand how it has been possible to apply special programs that allow the formation of competitive talent even though it has not been possible to increase the available resources, in a first comparative in the campus of Corregidora, Huimilpan, Menchaca, Montenegro, Pedro Escobedo, Querétaro and San Juan del Río.

Keywords: project-based learning, engineering bases, high school, STEM.

\section{Resumo}

O ensino médio (SGA) no México agora é considerado parte do ensino obrigatório no país. Diante dessa obrigação, o desenvolvimento de uma educação com maior impacto no ambiente produtivo e educacional se torna mais importante. A aprendizagem baseada em projetos e os estudos de caso provaram ser uma maneira de gerar aprendizagem significativa, embora desenvolvê-la com os escassos recursos disponíveis em instituições públicas do país represente um enorme desafio. Por outro lado, está comprovado que as habilidades vinculadas à abordagem científica integrada, a 


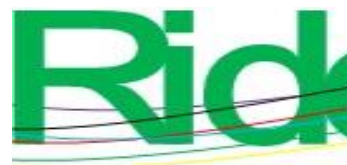

\section{Revista Iberoamericana para la Investigación y el Desarrollo Educativo \\ ISSN $2007-7467$}

saber: ciência, tecnologia, engenharia e matemática (STEM), são muito importantes na integração de uma aprendizagem mais ativa com Melhor compreensão do conteúdo científico. No SGA do México, existe o modelo tradicional de educação, treinamento técnico e o modelo dual (agora através do modelo de treinamento duplo mexicano); no entanto, embora sejam bons esforços, eles se concentram mais no ensino tradicional, no treinamento técnico ou na solução de problemas para ganhar experiência do que desenvolver uma capacidade de "engenhosidade" para resolver problemas práticos, mas não focados necessariamente trabalhar em uma empresa específica; capacidade que lhes permita resolver facilmente problemas e lidar melhor com compromissos, como lidar com o estudo da engenharia e, posteriormente, com diferentes tarefas de pesquisa aplicada.

Assim, com base no programa Project Lead The Way (PLTW), desenvolvido nos Estados Unidos, a Faculdade de Estudos Científicos e Tecnológicos de Querétaro (Cecyteq) desenvolveu um programa chamado Bases de Engenharia, que busca desenvolver no Habilidades dos alunos do EMS que lhes permitem enfrentar com mais êxito os estudos universitários de engenharia e o desenvolvimento de atividades profissionais em sua especialidade técnica no campo do trabalho. Este artigo mostra o monitoramento da eficiência do programa e a satisfação que ele causou nos alunos. O exposto acima, com o objetivo de entender como aplicar programas especiais que permitam a formação de talentos competitivos, apesar de não haver um aumento nos recursos materiais disponíveis, em uma primeira comparação nas Corregidora, Huimilpan, Menchaca, Montenegro, Pedro Escobedo, Querétaro e San Juan del Río.

Palavras-chave: aprendizagem baseada em projetos, bases de engenharia, ensino médio, STEM. Fecha Recepción: Mayo 2019 Fecha Aceptación: Diciembre 2019 


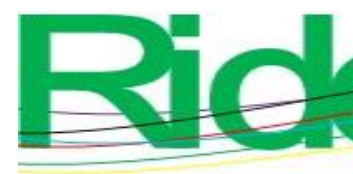

Revista Iberoamericana para la

Investigación y el Desarrollo Educativo

ISSN $2007-7467$

\section{Introducción}

De acuerdo con cifras del Instituto Nacional de Estadística, Geografía e Informática [Inegi] (2017), Querétaro es el quinto estado en México con mayor crecimiento, solo seguido de Aguascalientes, Baja California Sur, Quintana Roo y Yucatán. Y siguiendo datos de la Secretaría de Desarrollo Sustentable [Sedesu] (2015), se cuenta en el estado con 22 parques industriales o tecnológicos, teniendo como sectores estratégicos alimentos, bebidas, automotriz, electrodomésticos y aeroespacial. En un estado con una economía tan creciente, el desarrollo de la educación, y en particular la media superior, se vuelve crucial. Particularmente debido a que una parte de los egresados de dicho nivel se incorpora desde ese momento a la vida laboral, mientras que el resto decide seguir estudiando para obtener una licenciatura o ingeniería e incorporarse en el mercado laboral al término de esta.

A nivel nacional, la educación media superior, según el Instituto Nacional para la Evaluación de la Educación [INEE] (2014), tuvo el mayor aumento con 1.7 millones de alumnos, seguida de la educación superior con casi 1.4 millones. Por ejemplo, en el estado de Querétaro, el subsistema de los Colegios de Estudios Científicos y Tecnológicos del estado de Querétaro (Cecyteq) en los últimos seis años (2008-2015) creció más de $200 \%$ su matrícula de estudiantes. Actualmente, el Cecyteq es la primera opción de educación media superior en el estado mediante un modelo de bachillerato tecnológico bivalente vinculado con el sector productivo de la región, el cual debe ser fortalecido con la educación basada en ingeniería.

De acuerdo con Peña y Bermúdez (2016), la formación educativa se ve reforzada por la vinculación entre la empresa y las instituciones, lo cual permite que sea más significativo el aprendizaje cuando existe la posibilidad de desarrollar proyectos reales con un enfoque innovador. En un estudio conducido por Cu Balán (2005) sobre las trayectorias previas de los estudiantes de ingenierías en Campeche, se identificaron como principales causas del bajo desempeño la falta de orientación, de motivación y los conocimientos bajos del nivel medio superior, lo que muestra la importancia de esta preparación preliminar. Este no es un caso aislado, ya que ha sido analizado por Mares et al. (2012), Sosa, Barrientos, Castro y García (2010) y Sosa, Tuyub y Aparicio (2014), entre otros. La posibilidad que tiene un país de acceder a estudios superiores de desarrollo y bienestar depende del nivel escolar de su población y de su capacidad de resolver problemas e innovar. 


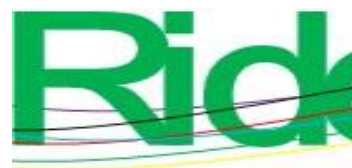

\section{Revista Iberoamericana para la Investigación y el Desarrollo Educativo \\ ISSN $2007-7467$}

González, González y Miles (2001) mencionan que adaptar la oferta educativa a las necesidades de mercado laboral ha sido un tema escasamente analizado, y que cuando ha sido analizado, ha sido de manera muy empírica. Bunk (1994), por su parte, aclara que una persona que tiene conocimientos, destrezas y actitudes que permiten que resuelva los problemas de forma autónoma y flexible posee una competencia profesional. Esto se logra en parte con los programas basados en ingeniería. Los estudios muestran que los países que han planeado su futuro dando la mayor prioridad a la cobertura y calidad de su sistema educativo, así como al desarrollo de la ciencia, la ingeniería y la tecnología, han logrado acceder a la sociedad del conocimiento (Navarro, Iglesias y Torres 2006). La formación del acervo de talento de estas disciplinas es una tarea estratégica para la nación y demanda un liderazgo visionario que tiene por resultado, en el mediano y largo plazo, llegar a niveles superiores de lo que ahora se denomina la economía del conocimiento.

Ahora bien, para García, Reyes y Burgos (2017) es importante considerar las habilidades ligadas a la ciencia, tecnología, ingeniería y matemática (STEM, por sus siglas en inglés). En esa misma línea, Becker y Park (2011) afirman que la integración de estas asignaturas tienen un efecto positivo en los estudiantes. Sin embargo, de acuerdo en esta ocasión con Vo, Zhu y Diep (2017), las disciplinas del STEM deben cumplir dos requisitos: 1) ser de los campos de la ciencia, tecnología, ingeniería y matemáticas y 2) clasificar dentro de uno de los cuatro grupos pertenecientes a las ciencias duras.

Para hablar mejor de las características que se buscan en los programas de ingeniería es importante mencionar que se buscan habilidades y destrezas basadas en la concepción de competencia. Para Sebastián (2012), la base de las competencias son los rasgos y características que ayudan a explicar el por qué la gente se inserta en diferentes experiencias y adquiere diferentes niveles de destrezas, habilidades y conocimientos, que son el segundo peldaño, el cual solo se alcanza mediante una serie de experiencias de aprendizaje para convertirse en competencias. Así, pues, todavía en los términos de Sebastián (2012), una competencia se puede usar de muchas maneras, pero esta adquiere utilidad en un contexto. En lo anterior radica el mayor reto: determinar qué competencias pueden agregarse para alcanzar una combinación óptima de destrezas, habilidades y conocimientos y crear tareas específicas. 


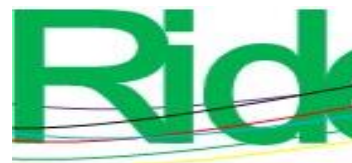

\section{Revista Iberoamericana para la Investigación y el Desarrollo Educativo \\ ISSN $2007-7467$}

En este panorama es donde se inserta la necesidad de desarrollar un programa surgido desde la educación media superior que permita, mediante la base de rasgos y características de dicho nivel educativo, generar experiencias de aprendizaje significativo para desarrollar destrezas y habilidades que posteriormente se integren y generen competencias afines a las necesidades del sector. En la figura 1 se puede observar que la base genera experiencias de aprendizaje que, sumadas a las destrezas, habilidades y el conocimiento que se transmiten, permiten, mediante experiencias de aprendizaje integradoras, la formación de competencias, y que la mejor manera de evaluar a estas es mediante las demostraciones, donde el aprendizaje basado en proyectos juega un papel crucial. De este programa se hablará más adelante.

Figura 1. Experiencias de aprendizaje significativo

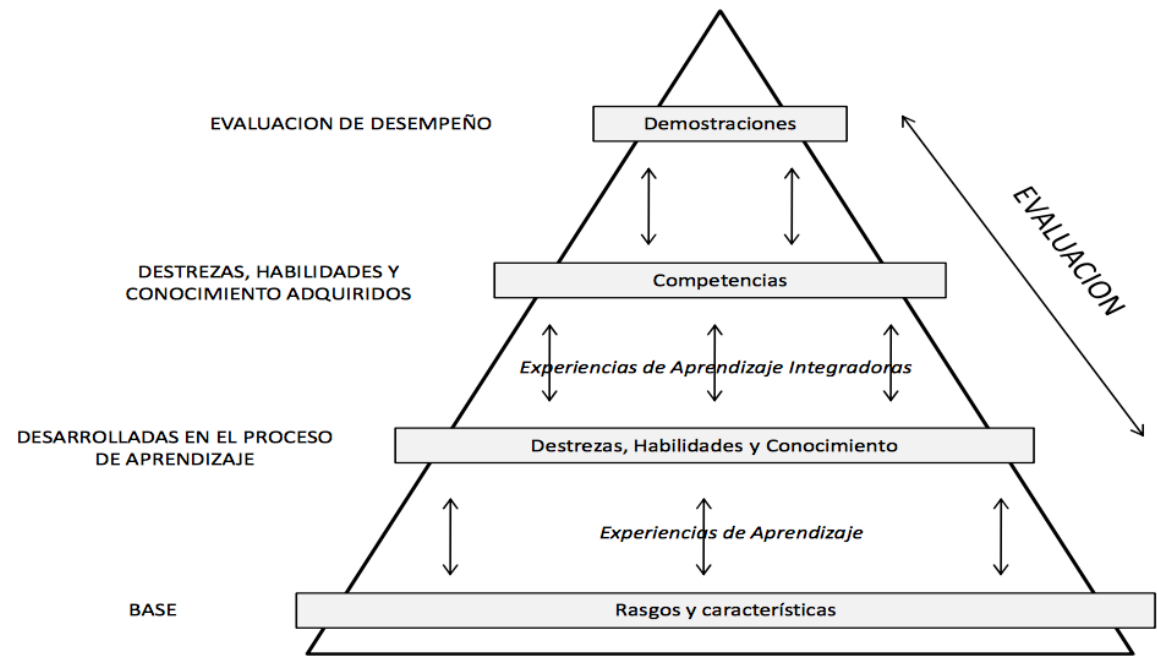

Fuente: Elaboración propia con base en el Centro de Estudios para la Enseñanza y Política del Departamento de Educación de Estados Unidos

De acuerdo con Morán (2012), el nivel de desarrollo de un país depende de la cantidad y calidad de los científicos e ingenieros con los que cuenta, pues son esenciales para la solución de los problemas del desarrollo, generar la infraestructura y las innovaciones en productos y servicios, incrementar la productividad, crear empleos bien remunerados, elevar la competitividad nacional y mejorar el bienestar general. El nivel de desempeño del acervo de ingenieros que tiene un país depende de su cantidad y, según Tobón, Rial, Carretero y García (2006), también de la calidad de las instituciones de educación superior en las que se formaron. 


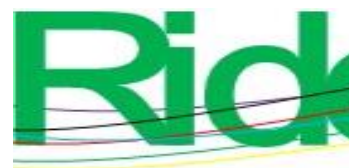

\section{Revista Iberoamericana para la Investigación y el Desarrollo Educativo ISSN $2007-7467$}

Bottoms y Uhn (2007), así como Blais y Adelson (1998), buscando dar respuesta a una problemática real de la región que tiene que ver con esa necesidad de formar más ingenieros y con mejores habilidades, analizaron el programa Project Lead the Way (PLTW), el cual nació en 1997 en Nueva York, Estados Unidos, y que mediante una secuencia de cursos que preparan a los estudiantes en las habilidades necesarias para las ingenierías, fomenta el desarrollo y el interés en las ciencias mediante seis metas estratégicas:

1) Incrementar el número de jóvenes que buscaban un programa de dos a cuatro años en las áreas de ingenierías.

2) Proveer de estándares claros y expectativas de éxito en el programa.

3) Proveer liderazgo y soporte basado en la mejora continua en la innovación.

4) Reducir las tasas de deserción escolar en las carreras de ingeniería.

5) Contribuir a la prosperidad del país.

6) El enfoque es un tercio teórico y dos tercios de práctica, tomando como enfoque principal de las clases la resolución de problemas basados en proyectos (Blais y Adelson, 1998).

El enfoque se tornó muy importante principalmente ante el preocupante descenso de los últimos años del interés de los jóvenes por el estudio del STEM, lo cual, en sintonía con lo dicho por Rocard, Csermely, Jorde, Walwerg-Henriksson y Hemmo (2007), se está traduciendo en un insuficiente número de técnicos y científicos que permita mantener de manera adecuada el desarrollo clave de los sistemas de ciencia y tecnología no solo en Europa sino del mundo entero. Además de lo anterior, Vázquez, de Talavera y Austin (2013) mencionan que los rasgos mejor valorados de la ciencia escolar son la utilidad para un trabajo futuro, ya que el aumentar la curiosidad para conocer permite interesarse por cosas que no se explican todavía y mejora las expectativas de carrera; y por el lado negativo, la dificultad percibida en el aprendizaje. 


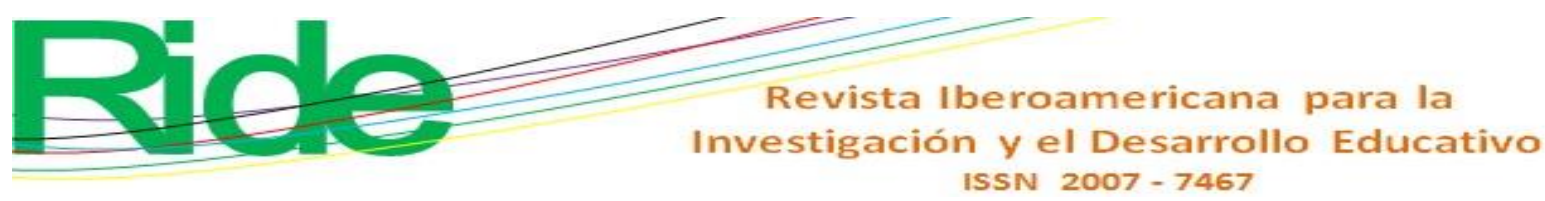

\section{Metodología}

Con todo lo anteriormente expuesto, la dirección del Cecyteq buscó generar un programa, con apoyo de la Fundación México-Estados Unidos para las Ciencias (Fumec), que estuviera adaptado a la realidad de nuestro país, la cual es muy diferente a las realidades de otros países desde el punto de vista primordialmente administrativo, ya que incorporar una currícula como la del programa Project Lead The Way (PLTW) implica un costo administrativo alto. Por lo que se implementó un proyecto adaptado a la realidad de nuestro país, como se decía, buscando solo impartirlo en una o dos horas más por día adicionales a los grupos tradicionales, con el fin de mantener el objetivo primordial de fomentar el interés por las ciencias y facilitar su estudio posterior sin comprometer la estabilidad financiera de la institución.

Figura 2. Programa de materias del proyecto Bases de Ingeniería
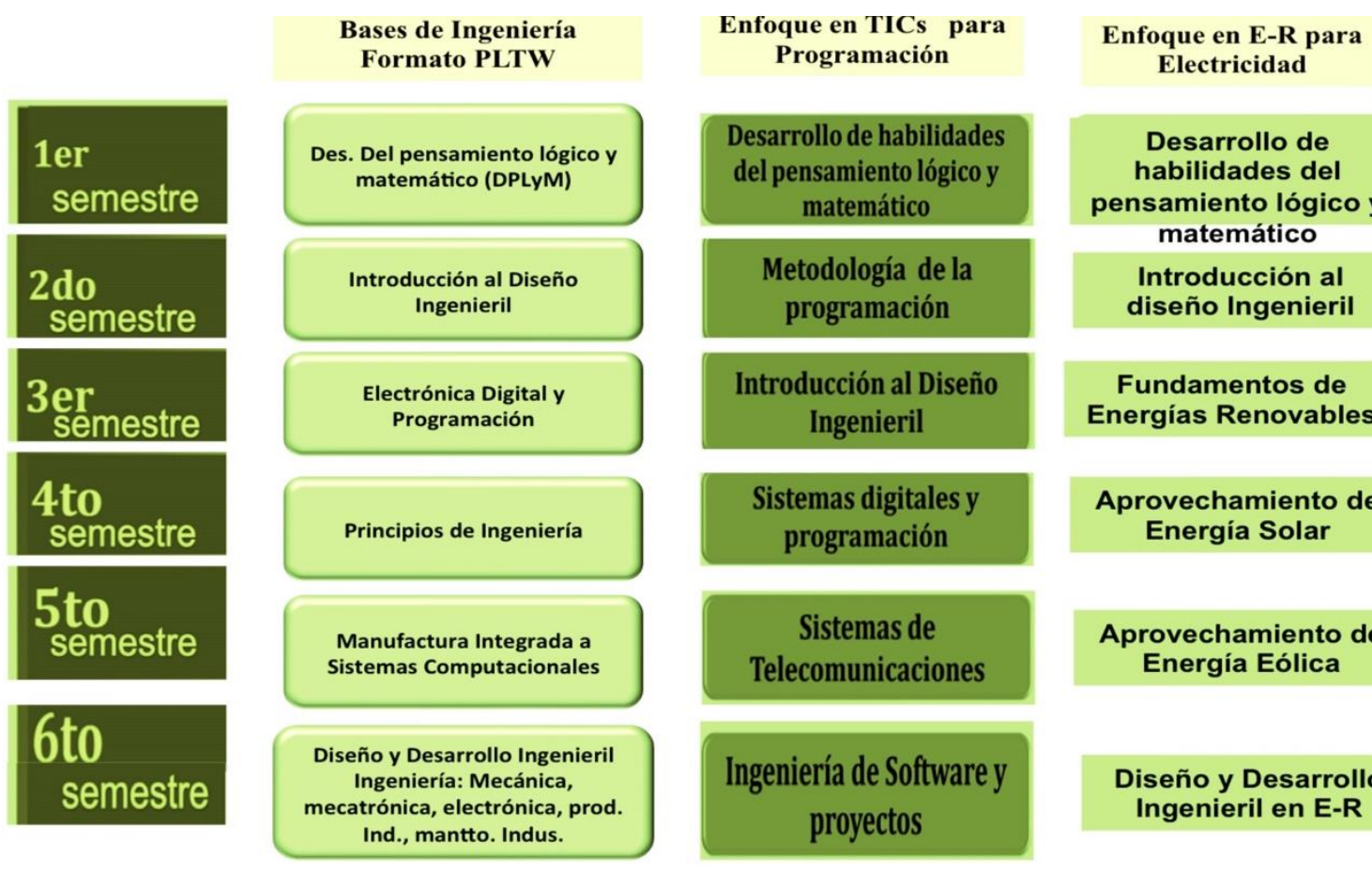

Fundamentos de Energias Renovables

Aprovechamiento de Energía Solar

Aprovechamiento de Energía Eólica

Diseño y Desarrollo Ingenieril en E-R

Fuente: Elaboración propia

Para desarrollar este estudio, se contempló dar seguimiento a los estudiantes que ingresaron a Bases de Ingeniería, como se denominó el programa, el cual se visualiza en la figura 2, en un periodo del 2009 al 2014, contemplando con esto las generaciones que egresaron en los periodos del 2012 al 2017. Los estudiantes fueron ingresando de manera gradual inicialmente solo en los planteles Querétaro y Corregidora, que son los que se encuentran más cercanos a la parte 


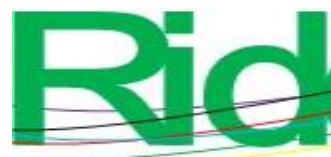

Revista Iberoamericana para la Investigación y el Desarrollo Educativo

ISSN $2007-7467$

metropolitana del estado de Querétaro; posteriormente, ya en 2011, se agregaron alumnos en San Juan del Río; en 2012, en Pedro Escobedo; 2013, en Huimilpan, y en 2015 en Menchaca y Montenegro hasta llegar a un total de 1500 alumnos en el programa.

Se identificaron varias propuestas de metodología para evaluar la satisfacción, tal y como la de Llarena y Páparo (2006) y la de Douglas, Douglas y Barnes (2006), entre otras, pero nos encontramos con la problemática de que en muchas ocasiones son en inglés o adaptadas a un contexto diferente, además de no medir lo que buscábamos medir. Con base en lo anterior nos enfrentamos ante la disyuntiva de emplearlo de manera directa o no, y revisando a Hernández, Fernández y Baptista (2010) encontramos que en ocasiones los instrumentos desarrollados en el extranjero y que no han sido validados para nuestro contexto principalmente en tiempo y cultura no siempre es lo más adecuado. Traducir el instrumento no equivale a validar el mismo, aun cuando se traduzca adaptándolo al tipo de lenguaje, por lo que se optó por desarrollar un instrumento y hacerlo en una pequeña población piloto con la finalidad de validarlo para el estudio en cuestión.

Si bien el programa puede ser implementado en diferentes carreras, ya que, como se mencionó anteriormente, el modelo del Cecyteq es de bachillerato bivalente, es decir, mientras el alumno estudia la preparatoria obtiene a la vez una carrera técnica. En un inicio las carreras en las que se maneja el modelo del programa Bases de Ingeniería fueron las siguientes: Programación, Mecatrónica, Informática, Mantenimiento Industrial, Producción Industrial y Electrónica. La figura tres ilustra a continuación la ubicación geográfica de los planteles de Cecyteq. 


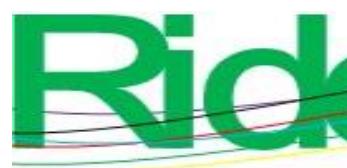

\section{Revista Iberoamericana para la Investigación y el Desarrollo Educativo ISSN $2007-7467$}

Figura 3. Ubicación de los planteles de Cecyteq y los programas Bases de Ingeniería

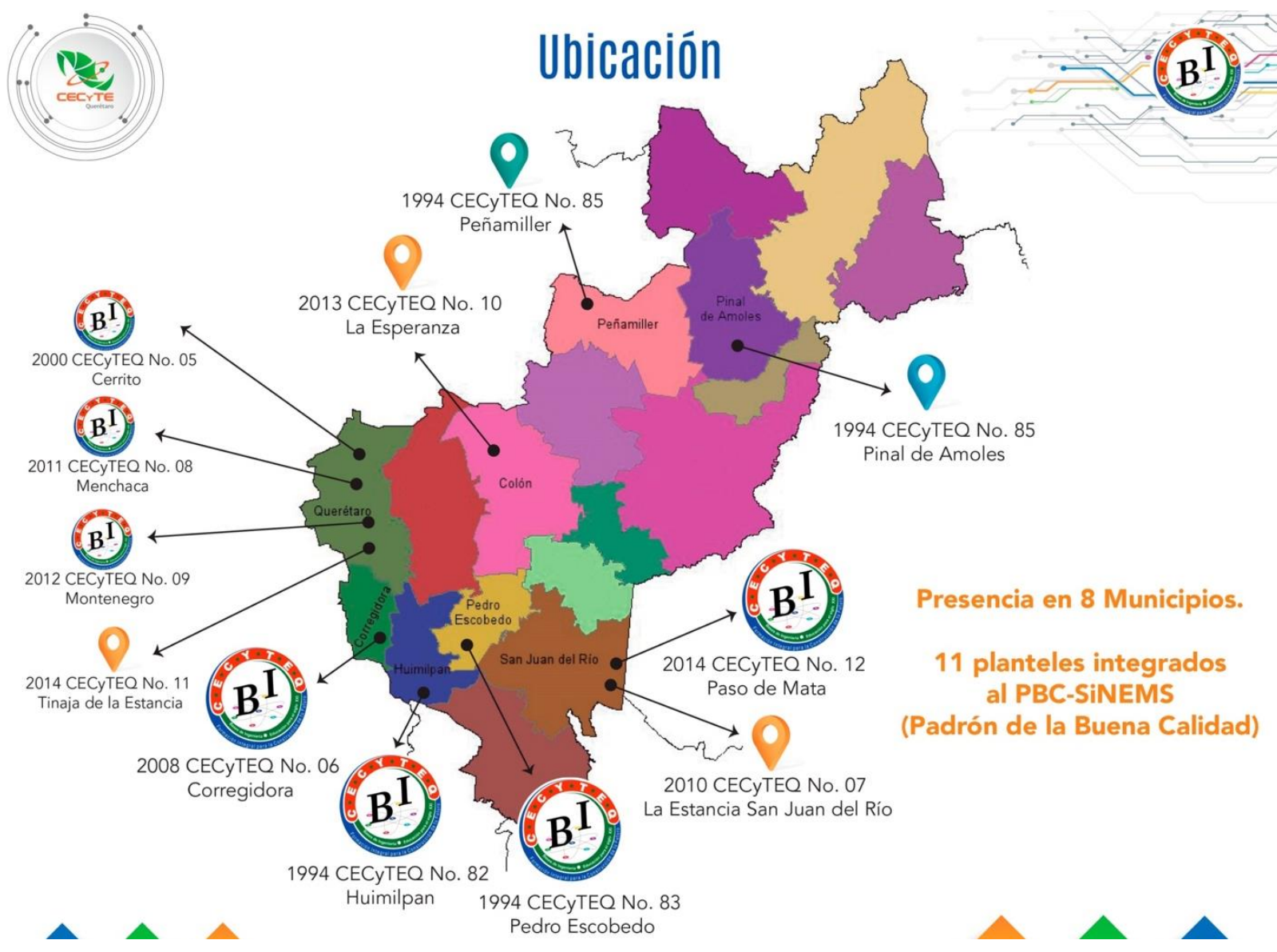

Fuente: Elaboración propia con base en Cecyteq

Para seleccionar a los alumnos, se aplicó una encuesta donde manifestaban el interés de participar en el programa. Luego se registró el total de alumnos que ingresaron al programa de acuerdo con cada plantel y con la carrera elegida; se les dio seguimiento hasta el término del programa, y se restó el número de estudiantes que concluyeron con éxito el programa de estudios, tal y como se muestra en la tabla 1.

En la tabla 1 se muestran únicamente los ingresos de estudiantes al primer semestre y no aquellos que entraron posteriormente, ya que los que ingresos en fechas posteriores son muy pocos y usualmente son reemplazos a los que ya se encontraban adentro. Los que egresaron incluyen el descuento de los que se cambiaron a programa tradicional y aquellos estudiantes considerados como irregulares, es decir, que seguían con materias aún pendientes. 


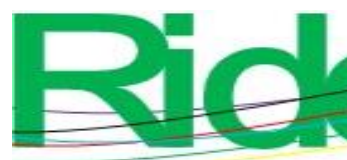

Revista Iberoamericana para la Investigación y el Desarrollo Educativo ISSN $2007-7467$

Tabla 1. Ingresos a Bases de Ingeniería 2009-2014

\begin{tabular}{|c|c|c|c|c|c|c|c|}
\hline \multicolumn{8}{|c|}{ Generaciones de Bases de Ingeniería } \\
\hline & & $\begin{array}{l}2014- \\
2017\end{array}$ & $\begin{array}{l}2013- \\
2016\end{array}$ & $\begin{array}{l}2012- \\
2015\end{array}$ & $\begin{array}{l}2011- \\
2014\end{array}$ & $\begin{array}{l}2010- \\
2013\end{array}$ & $\begin{array}{l}2009- \\
2012\end{array}$ \\
\hline Plantel & Carrera & $\begin{array}{c}\text { Entraro } \\
\text { n en } \\
\text { 1er. } \\
\text { Semestr } \\
\text { e }\end{array}$ & $\begin{array}{c}\text { Entraro } \\
\text { n en } \\
\text { 1er. } \\
\text { Semestr } \\
\text { e }\end{array}$ & $\begin{array}{c}\text { Entraro } \\
\text { n en } \\
\text { 1er. } \\
\text { Semestr } \\
\text { e }\end{array}$ & $\begin{array}{c}\text { Entraro } \\
\text { n en } \\
\text { 1er. } \\
\text { Semestr } \\
\text { e }\end{array}$ & $\begin{array}{c}\text { Entraro } \\
\text { n en } \\
\text { 1er. } \\
\text { Semestr } \\
\text { e }\end{array}$ & $\begin{array}{c}\text { Entraro } \\
\text { n en } \\
\text { 1er. } \\
\text { Semestr } \\
\text { e }\end{array}$ \\
\hline $\begin{array}{c}\text { Corregidora } \\
(06)\end{array}$ & Programación & 36 & 36 & 35 & 38 & & \\
\hline $\begin{array}{c}\text { Corregidora } \\
(06)\end{array}$ & Mecatrónica & 72 & 35 & 36 & 36 & 35 & \\
\hline $\begin{array}{c}\text { Corregidora } \\
\text { (06) }\end{array}$ & Informática & & & & & 36 & 48 \\
\hline $\begin{array}{c}\text { Huimilpan } \\
\text { (82) }\end{array}$ & $\begin{array}{c}\text { Mantenimient } \\
\text { o Industrial }\end{array}$ & 35 & 35 & & & & \\
\hline $\begin{array}{c}\text { Menchaca } \\
(08)\end{array}$ & $\begin{array}{l}\text { Producción } \\
\text { Industrial }\end{array}$ & 30 & & & & & \\
\hline $\begin{array}{c}\text { Montenegro } \\
(09)\end{array}$ & Mecatrónica & 35 & & & & & \\
\hline $\begin{array}{c}\text { Pedro } \\
\text { Escobedo } \\
\text { (83) }\end{array}$ & Electricidad & 35 & 35 & 35 & & & \\
\hline $\begin{array}{c}\text { Pedro } \\
\text { Escobedo } \\
(83)\end{array}$ & Programación & 35 & 39 & & & & \\
\hline $\begin{array}{c}\text { Querétaro } \\
(05)\end{array}$ & Informática & & & & & 35 & 35 \\
\hline $\begin{array}{c}\text { Querétaro(05 } \\
\text { ) }\end{array}$ & Electrónica & 36 & 35 & 36 & 35 & 35 & 31 \\
\hline
\end{tabular}




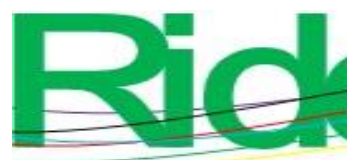

Revista Iberoamericana para la Investigación y el Desarrollo Educativo

ISSN $2007-7467$

\begin{tabular}{|c|c|c|c|c|c|c|c|}
\hline $\begin{array}{c}\text { Querétaro(05 } \\
\text { ) }\end{array}$ & $\begin{array}{c}\text { Mantenimient } \\
\text { o }\end{array}$ & 36 & 35 & 35 & 35 & 35 & 32 \\
\hline $\begin{array}{c}\text { Querétaro(05 } \\
\text { ) }\end{array}$ & Programación & 36 & 35 & 36 & 34 & & \\
\hline $\begin{array}{c}\text { San Juan del } \\
\text { Río (07) }\end{array}$ & $\begin{array}{c}\text { Producción } \\
\text { Industrial }\end{array}$ & 32 & 34 & 35 & 16 & & \\
\hline & Total & 418 & 319 & 248 & 194 & 176 & 146 \\
\hline
\end{tabular}

Fuente: Elaboración propia con base en datos de Control Escolar del Cecyteq

\section{Resultados}

Del total de ingresos, la distribución no fue uniforme en parte debido a que los planteles, como se mencionó anteriormente, se han ido añadiendo a lo largo del tiempo e incorporando grupos al programa en cuestión, si bien la eficiencia terminal por generación sube y baja como se muestra en la tabla 2. También es importante analizar a detalle si este grado de deserción se debe a los planteles, las generaciones, las carreras o simplemente asuntos generales relacionados con los mecanismos de retención de los estudiantes. En la tabla 3 se observa el desglose de la eficiencia terminal por plantel.

Tabla 2. Eficiencia terminal por generación

\begin{tabular}{|c|c|}
\hline Generación & Eficiencia terminal \\
\hline $2009-2012$ & $76 \%$ \\
\hline $2010-2013$ & $74 \%$ \\
\hline $2011-2014$ & $89 \%$ \\
\hline $2012-2015$ & $72 \%$ \\
\hline $2013-2016$ & $85 \%$ \\
\hline $2014-2017$ & $77 \%$ \\
\hline
\end{tabular}

Fuente: Elaboración propia con base en Cecyteq 


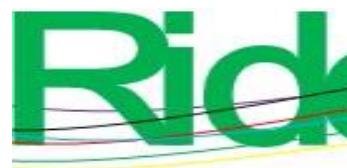

Revista Iberoamericana para la Investigación y el Desarrollo Educativo

ISSN $2007-7467$

Tabla 3. Eficiencia terminal por plantel del programa Bases de Ingeniería

\begin{tabular}{|l|r|}
\hline \multicolumn{1}{|c|}{ Plantel } & Eficiencia terminal \\
\hline Corregidora & $83 \%$ \\
\hline Huimilpan & $63 \%$ \\
\hline Menchaca & $50 \%$ \\
\hline Montenegro & $83 \%$ \\
\hline Pedro Escobedo & $77 \%$ \\
\hline Querétaro & $79 \%$ \\
\hline San Juan del Rio & $80 \%$ \\
\hline
\end{tabular}

Fuente: Elaboración propia con base en Cecyteq

Sin embargo, al revisar los indicadores por plantel podemos ver que hay dos planteles en los cuales la eficiencia terminal ha sido muy baja. Los resultados de manera general nos permiten ver información importante como la siguiente:

Antes que nada, $66 \%$ de los encuestados son hombres, mientras que $34 \%$ son mujeres, lo que implica que sigue existiendo preferencia por las habilidades desarrolladas por el STEM en los varones. Respecto a lo anterior, podemos ver que los resultados obtenidos en el trabajo de las generaciones del programa Bases de Ingeniería nos muestran un incremento en el interés de los estudiantes, en donde $96.1 \%$ de los alumnos del programa desean seguir estudiando una carrera, $\mathrm{y}$ de estos al menos $62 \%$ busca que sea en una ingeniería; tan solo $3.9 \%$ no planean seguir estudiando o piensan insertarse en el mercado laboral. De estos, el impacto directo en el semestre inmediato es de $79.5 \%$ que desea ingresar inmediatamente, $8.3 \%$ planea trabajar y después ingresar a la universidad y $8.8 \%$ planea esperar entre seis meses y un año antes de ingresar (véase figura 4. Planes al finalizar el bachillerato). 


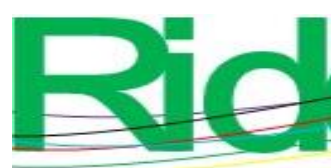

\section{Revista Iberoamericana para la Investigación y el Desarrollo Educativo ISSN $2007-7467$}

Figura 4. Planes al finalizar el bachillerato

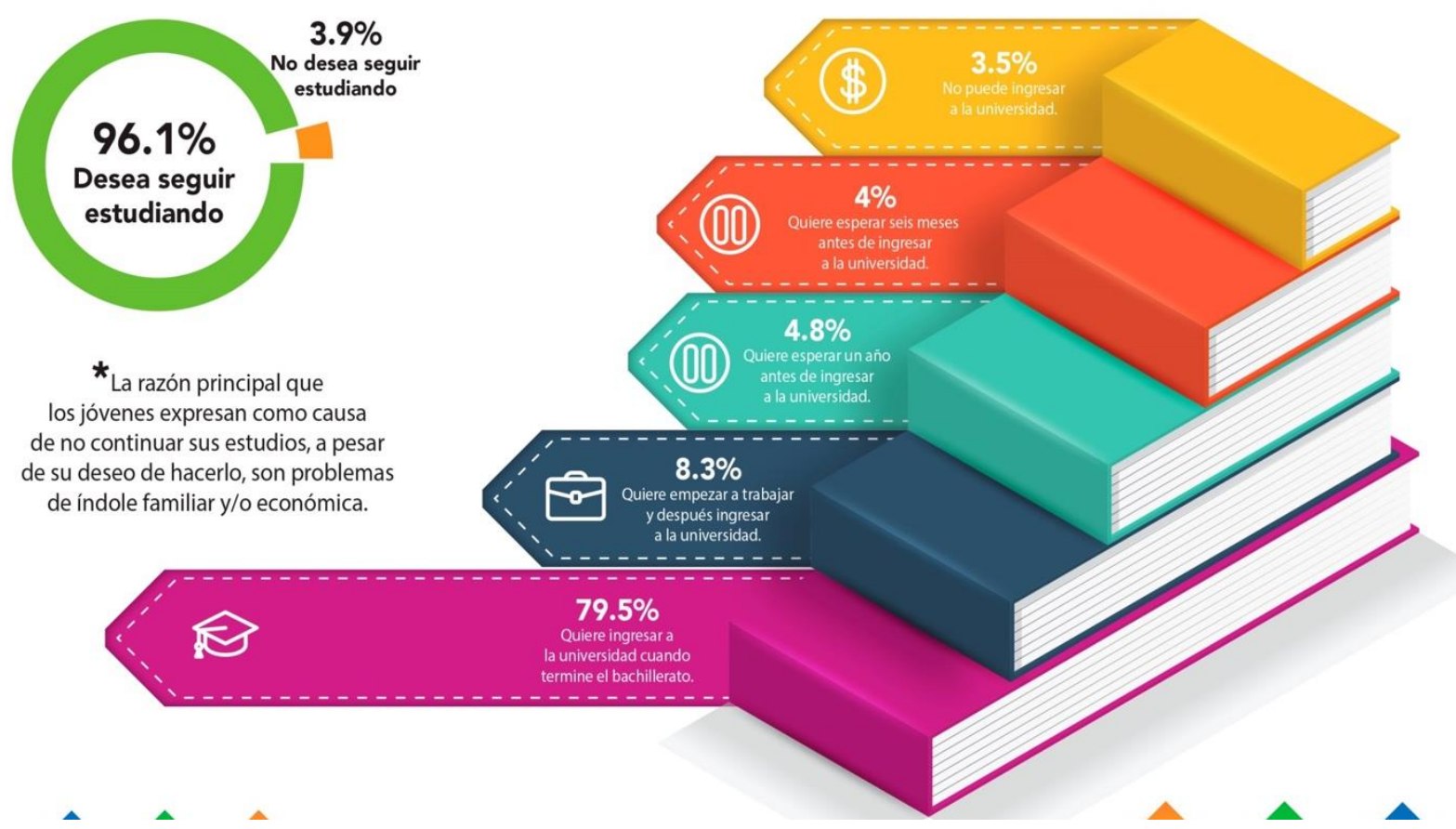

Fuente: Elaboración propia con base en Cecyteq

Otros datos interesantes son que, a pesar de decir que las materias más difíciles son las relacionadas con matemáticas, física e inglés, más de $75 \%$ de los alumnos considera que son sus materias favoritas, lo que muestra el impacto de enseñar materias como estas mediante el aprendizaje basado en proyectos, es decir, que sea factible que a pesar de que no las encuentren sencillas les gusten. Otro dato que resultó bastante relevante es que los alumnos del programa presentan menos impacto del nivel socioeconómico de la familia respecto del grado de deserción, es decir, desertan menos que los alumnos de los esquemas tradicionales a pesar de provenir de un entorno socioeconómico bajo.

Una vez terminado el programa, a pesar de las dificultades para continuar el seguimiento a 413 alumnos egresados, se obtuvo como resultado que $80 \%$ continuaron sus estudios. De estos, 73 $\%$ eligieron carreras de ingenierías o ciencias y continuaron sus estudios en las principales instituciones del estado, el Instituto Tecnológico de Querétaro (ITQ) - hoy Tecnológico Nacional-, la Universidad Politécnica de Querétaro (UPQ), la Universidad Tecnológica de Querétaro (UTEQ) y la Universidad Autónoma de Querétaro (UAQ), principalmente (ver figuras 5 у 6). 
Figura 5. Alumnos que continúan en ingeniería o ciencias
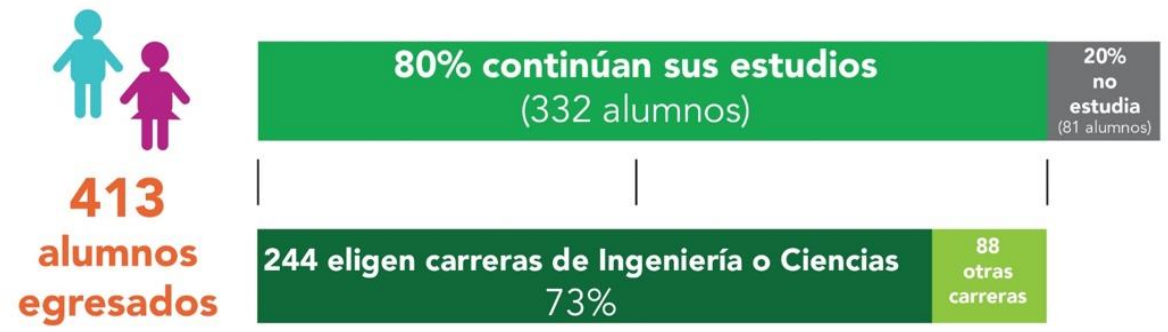

Fuente: Elaboración propia con base en Cecyteq

Figura 6. Preferencias en la elección de universidades

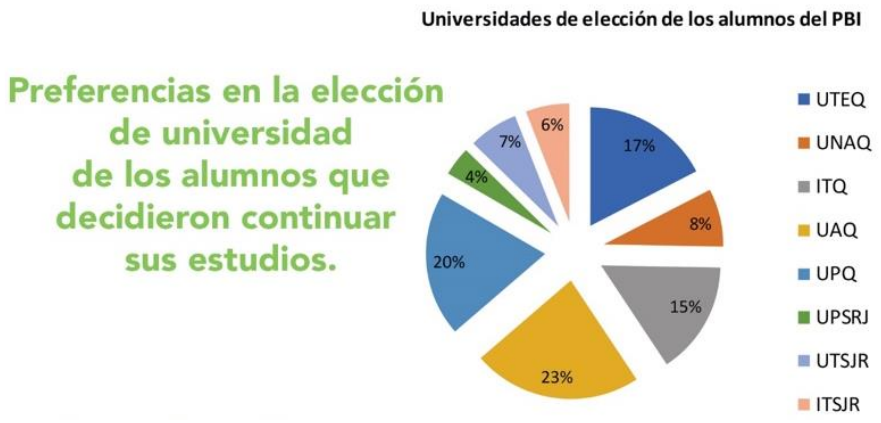

Fuente: Elaboración propia con base en Cecyteq

\section{Discusión}

Al inicio de la investigación se planteaba cómo promover mediante el impulso al desarrollo de las habilidades de STEM las habilidades del pensamiento, y permitir que los estudiantes puedan generar mejores condiciones para que continúen de la educación media superior a la educación superior con un tránsito adecuado, y que se incremente no solo el número de alumnos que tienen gusto por las carreras de ingenierías, tecnologías o ciencias, sino también el número de alumnos que continúen estudiando - y esto les ofrezca mayores probabilidades de éxito en la conclusión de sus estudios.

$\mathrm{Al}$ inicio se revisaron diversos autores y publicaciones que mostraban resultados sobre cómo ha funcionado de manera importante programa PLTW en Estados Unidos; sin embargo, no había una adecuación similar para México, una en donde se pudiera analizar el impacto que tiene el desarrollar estos programas, principalmente en la educación media superior bivalente, que es la 


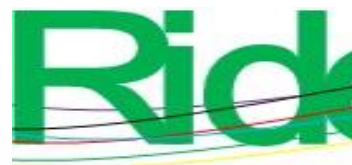

Revista Iberoamericana para la Investigación y el Desarrollo Educativo

ISSN $2007-7467$

que presenta las posibilidades tanto de profundizar en las competencias genéricas como en las disciplinares. En suma, resultados muy similares a los esperados al revisar que los autores nos hablan de la importancia del desarrollo de estas habilidades como una manera de impulsar el conocimiento y el incremento de ingenieros en los países sin generar programas muy costosos que pongan en riesgo el presupuesto.

En conclusión, los esfuerzos ligados a fortalecer las habilidades ligadas al STEM permiten crear en los estudiantes de educación media superior una forma de pensamiento que les resolver problemas e incrementar sus posibilidades de continuar estudiando una carrera ligada con las ingenierías, tecnología o las ciencias, las cuales no solo son necesarias en países como México, sino parte importante del pilar de disminuir la dependencia tecnológica. El presente trabajo muestra, además, que si bien es cierto que se sigue considerando que estas áreas "duras" no son fáciles, el ver y aprender desde una perspectiva basada en proyectos permite que se vean menos áridas y se motive a los alumnos a realizar proyectos aunque sean complejos. Este artículo muestra de manera general este seguimiento por varios años en diferentes planteles con condiciones diferentes socioeconómicas a lo largo de todo el estado de Querétaro, pero con un común resultado, que es la importancia que tiene el programa Bases de Ingeniería en impulsar el desarrollo de los jóvenes y sus posibilidades de integrarse en las áreas de ingeniería, tecnología y ciencias.

\section{Conclusiones}

El programa Bases de Ingeniería presenta una alternativa importante muy adecuada para el desarrollo de las habilidades ligadas al STEM; propicia una mejora en la resolución de los problemas y se muestra como una alternativa de bajo costo, tangible de replicar los esfuerzos de iniciativas como el PLTW en Estados Unidos.

Si bien es importante continuar dando seguimiento a las generaciones de alumnos, los datos de las generaciones aquí analizadas muestran un número interesante de estudiantes que continúan su preparación en la educación superior, así como un incremento en las posibilidades de ser admitidos y continuar sus estudios principalmente en las áreas de ingeniería y tecnología. 


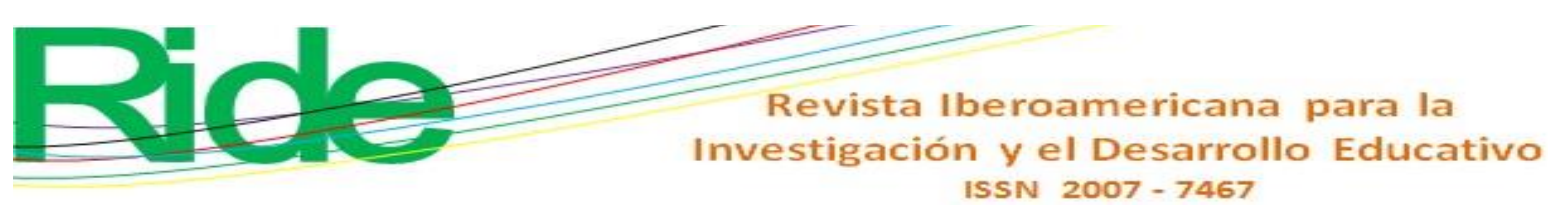

estudiantes de Psicología en la FES Iztacala. Enseñanza e Investigación en Psicología, 17(1).

Morán, C. A. (2012). Estado del arte y prospectiva de la ingeniería en México y el mundo. Informe final de la Academia de Ingeniería al Consejo Nacional de Ciencia y Tecnología (Conacyt).

Navarro, M. M., Iglesias, M. P. y Torres, P. R. (2006). Las competencias profesionales demandadas por las empresas: el caso de los ingenieros. Revista de Educación, 341, 643-661.

Peña, J. M. y Bermúdez, C. P. (2016). Vinculación universidad empresa. Innovación para la diversificación de mercados en cacao. Revista Iberoamericana de Contaduría, Economía y Administración, 4(7), 103-121.

Rocard, M., Csermely, P., Jorde, D., Walwerg-Henriksson, H. and Hemmo, V. (2007). Science Education Now: A Renewed Pedagogy for the Future or Europe. Brussels, Belgium: European Commission.

Sebastián, J. C. (2012). Las orientaciones de la formación de los profesores de la educación superior para el siglo XXI en México. Cuadernos de Educación y Desarrollo, 1(30).

Sosa, R. C., Barrientos, L. G., Castro, E. G. and García, J. H. (2010). Academic performance, school desertion and emotional paradigm in university students. Contemporary Issues in Education Research, 3(7), 25.

Sosa, L., Tuyub, I. y Aparicio, E. (2014). Diagnóstico en estudiantes de nuevo ingreso a nivel superior: competencias y dificultades matemáticas. Ciudad de México, México: Comité Latinoamericano de Matemática Educativa.

Tobón, S., Rial, A., Carretero, M. y García, J. (2006). Competencias, calidad y educación superior. Ciudad de México, México: Editorial Magisterio.

Vázquez, Á., de Talavera, M. M. y Austin, I. (2013). Factores que influyen sobre la elección de estudios superiores de Ciencias y Tecnología. Enseñanza de las Ciencias: Revista de Investigación y Experiencias Didácticas, (Extra), 3642-3648.

Vo, H. M., Zhu, C. and Diep, N. A. (2017). The effect of blended learning on student performance at course-level in higher education: A meta-analysis. Studies in Educational Evaluation, $53,17-28$. 


\begin{tabular}{|l|l|}
\hline Rol de Contribución & $\begin{array}{c}\text { Revista Iberoamericana para la } \\
\text { Investigación y el Desarrollo Educativo } \\
\text { ISSN } 2007-7467\end{array}$ \\
\hline Conceptualización & Lutor (es) \\
\hline Metodología & Christian Paulina Mendoza Torres. \\
\hline Software & NO APLICA \\
\hline Validación & Juan Manuel Peña Aguilar. \\
\hline Análisis Formal & Juan Manuel Peña Aguilar. \\
\hline Investigación & Luis Fernando Pantoja Amaro \\
\hline Recursos & Luis Fernando Pantoja Amaro \\
\hline Curación de datos & Christian Paulina Mendoza Torres. \\
\hline $\begin{array}{l}\text { Escritura - Preparación del } \\
\text { borrador original }\end{array}$ & Juan Manuel Peña Aguilar. \\
\hline $\begin{array}{l}\text { Escritura - Revisión y } \\
\text { edición }\end{array}$ & Christian Paulina Mendoza Torres. \\
\hline Visualización & Juan Manuel Peña Aguilar. \\
\hline Supervisión & Luis Fernando Pantoja Amaro \\
\hline Administración de Proyectos & Juan Manuel Peña Aguilar. \\
\hline Adquisición de fondos & NO APLICA \\
\hline
\end{tabular}

